\title{
El celecoxib redujo los pólipos en los pacientes con polipomatosis adenomatosa familiar
}

The Effect of Celecoxib, a Cyclooxygenase-2 Inhibitor, in Familial Adenomatous Polyposis.

Gideon Steinbach, M.D., Phd., Patrick M Lynch, M.D.,J.D., Robin K.S.Phillips, M.B., B.S. N Eng J Med 2000;342:1946-52

\begin{abstract}
Objetivo
Evaluar los efectos quimio-preventivos del celecoxib (un inhibidor selectivo de la cox 2), sobre la progresión de los pólipos colónicos a cáncer, en pacientes con polipomatosis adenomatosa familiar (PAF).
\end{abstract}

\section{Diseño}

Estudio randomizado, doble ciego, controlado.

\section{Lugar}

Anderson Cancer Center, Houston, USA y St. Mark's Hospital, London, UK.

\section{Pacientes}

Se randomizaron 77 pacientes, hombres y mujeres entre 18 y 65 años, que presentaran al menos 5 pólipos de $2 \mathrm{~mm}$ o más y que no hubieran sido sometidos a recto-colectomía total. Se los siguió durante 6 meses. Criterios de exclusión:

- Historia de colectomía en los previos 12 meses, o programada para los 8 meses siguientes a la randomización.

- Uso de anti- inflamatorios no esteroides (AINES) 3 o más veces por semana dentro de los 6 meses de la randomización o de 102 veces por semana dentro de los 3 meses de la randomización.

- Pacientes con hemograma, función hepática o renal anormales.

\section{Intervención}

Los pacientes fueron randomizados en tres ramas: $100 \mathrm{mg}$. de celecoxib 2 veces por día (30), 400 mg. de celecoxib 2 veces por día (32) y placebo 2 veces por día, durante 6 meses (17). Fueron evaluados al inicio y a los 6 meses con: historia clínica, examen físico, y endoscopía con toma biopsias múltiples. En la evaluación inicial se testeó la presencia de la mutación del gen Apc (adenomatous polyposis coli). En la endoscopía inicial se realizó una marcación con tinta china en las áreas de mayor densidad de pólipos, para la evaluación posterior. Se compararon ambas endoscopias a través de parámetros cuantitativos (tamaño y numero de los pólipos) a través de fotografías y cualitativos (peor, iqual o mejor), a través de video-tapes. Se controló la adherencia mediante el recuento de píldoras de cada paciente.

\section{Medición de resultados principales}

\section{Reducción cuantitativa de los pólipos:}

- Porcentaje de reducción en el numero de pólipos respecto del basal. - Porcentaje de reducción de la carga polipoidea respecto del basal (suma del diámetro de todos los pólipos del área marcada con tinta). Se calculó la media de dichos porcentajes de reducción en cada una de los tres grupos. La eficacia fue medida comparando la media de descenso entre las ramas de tratamiento y la rama placebo y mediante análisis multivariado ajustando por: numero basal de pólipos, sexo, edad, sitio de estudio y estatus quirúrgico previo.

\section{Reducción cualitativa de los pólipos:}

- Determinación de un score: "mejor", "iqual" o "peor" de la endoscopía de control respecto de la basal. La media de dicho score para cada grupo fue comparada entre las ramas de tratamiento con la rama placebo.

\section{Resultados principales}

Se analizó por intención a tratar. Los grupos fueron homogéneos excepto en la edad, siendo más jóvenes los pacientes que recibieron celecoxib $400 \mathrm{mg}$. La mutación del gen Apc estuvo presente en 66 pacientes y otros 2 pacientes tenían familiares que la presentaban. Completaron el tratamiento 72 pacientes. Más del $90 \%$ de los pacientes que completaron el estudio tomaron al menos el $80 \%$ de la medicación. No hubo diferencias significativas en el numero de pólipos basales entre los tres grupos de tratamiento: $11.5 \pm 8.5,12.3 \pm$ 8.2 y $15.5 \pm 13.4$ para los grupos, Celecoxib 100, Celecoxib 400 y placebo respectivamente $(p=0.66)$.

Tanto los resultados cuanti como cualitativos mostraron diferencias significativas con el grupo Celecoxib 400, no observándose diferencias significativas entre el grupo Celecoxib 100 y el grupo placebo. El análisis multivariado confirmó la misma diferencia.

\begin{tabular}{l|c|c|c}
\hline \multicolumn{1}{c|}{ Variables } & Placebo & Celecoxib 100 & Celecoxib 400 \\
\hline $\begin{array}{l}\text { Porcentaje de cambio } \\
\text { en el numero de pólipos }\end{array}$ & $-4.5 \pm 16.4 \%$ & $-11.9 \pm 30.3 \%$ & $-28 \pm 24.0 \%$ \\
\hline \begin{tabular}{l} 
Valor de $p$ \\
\hline $\begin{array}{l}\text { Porcentaje de cambio } \\
\text { en la carga polipoidea }\end{array}$
\end{tabular} & $-4.9 \pm 17.3 \%$ & $-14.6 \pm 31.7 \%$ & $-30.7 \pm 25.7 \%$ \\
\hline $\begin{array}{l}\text { Valor de p } \\
\text { Media del score cualit. }\end{array}$ & $-0.07 \pm 0.26$ & $0.13 \pm 0.22$ & $0.33 \pm 0.32$ \\
\hline Valor de p & & 0.03 & $<0.001$ \\
\hline
\end{tabular}

Los valores de $\mathrm{p}$ son el resultado de la comparación de cada rama de tratamiento con el grupo placebo. Score cualitativo: $-1=$ peor; $0=$ igual; $1=$ mejor. La media del score cualitativo: es el promedio de los scores de los pacientes de cada rama del estudio.

Los efectos adversos más reportados fueron diarrea y dolor abdominal, sin diferencias significativas entre los 3 grupos.

\section{Conclusiones}

El celecoxib utilizado en dosis de $400 \mathrm{mg}$. dos veces por día, produce regresión de los pólipos colorectales de manera significativa en pacientes con polipomatosis adenomatosa familiar.

Fuente de financiamiento: The National Cancer Institute, Cancer Center Support Grant y Searle Pharmaceutics.

\section{COMENTARIO}

Considerando la magnitud de la polipomatosis adenomatosa familiar, en cuanto a su evolución en casi un $100 \%$ de los casos a cáncer y la gran morbimortalidad que ésta y su tratamiento acarrean, resulta interesante considerar los posibles efectos quimio-preventivos que podrían brindar los inhibidores de la COX2. Ya hay evidencia en la literatura de regresión de los pólipos con otros AINES, pero los estudios también presentan corto tiempo de seguimiento y su toxicidad a largo plazo dificultaría el uso crónico en la prevención del cáncer. En este estudio, la inhibición de la cox 2, lograría la regresión de un porcentaje significativo de pólipos. Sin embargo no existe evidencia clara en cuanto a si el tratamiento a largo plazo con este tipo de medicación

\section{Dra. Claudia Epelbaum}

Unidad de Medicina Familiar y Preventiva. Hospital Italiano de Buenos Aires.

1. Labayle D, Fischer D, Vielh P el al. Sulindac causes regression of rectal polyps in familial adenomatous polyposis. Gastroenterology 1991; $101: 635-9$.

2. Oshima M, Dinchuk JE, Kargman SL et al. Suppression of intestinal polyposis in Apc delta 716 knockout mice by inhibition of cyclooxygenase 2 (C0X2) Cell 1996: 87: 803-9. 3. Kawamori T, Rao CV, Seibert K, et al. Chemopreventive activity of celecoxib, a specific cyclooxigenase-2 inhibitor, against colon carcinogenesis. Cancer Re 1998; $58: 409-12$

puede evitar la progresión a cáncer o bien reemplazair, retrasar o limitar la extensión de la proctocolectomía a la que la mayoría de los pacientes con PAF serán sometidos en mayor o menor plazo. Por otro lado en este estudio no se evalúa el índice de recurrencia de los pólpos tras la suspensión del tratamiento. Sin embargo con los hallazgos actuales podría sugerirse la utilidad de los inhibidores de la cox 2 como terapia adyuvante para la supresión de la formación de pólipos en pacientes con área rectal residual tras la colectomía o bien en el período en el que el paciente esta aquardando la realización de una colectomía. A su vez estos estudios podrían ser el punto de partida de otros que evalúen la prevención de tumores colorectales en población de riesgo, incluyendo quienes presentan pólipos adenomatosos esporádicos. 